\title{
A case of papillary thyroid carcinoma discovered by elastography
}

\begin{abstract}
We report a case of papillary thyroid carcinoma (PTC) discovered by elastography (E). A 54years old female with a studied thyroid nodule during 5years, was performed E. This study shown an inner area of stiffness, that was punctured. The cytology reported suspicious of papillary carcinoma. The E improves the diagnostic performance of ultrasound (US). It is an additional tool that helps guide fine-needle aspiration (FNA) and estimates preoperative malignancy risk.
\end{abstract}

Keywords: elastography, papillary thyroid carcinoma
Volume 3 Issue 3 - 2017

Ivelís M Sarachi

Department of Breast Imaging and Women Care, Centro

Diagnóstico Mon, Argentina

\begin{abstract}
Correspondence: Ivelís M Sarachi, Department of Breast Imaging and Women Care, Centro Diagnóstico Mon, 7 street No. 1486, La Plata, Buenos Aires, Argentina, Tel +54221523528I, +542214392100, Email isarachi@diagnosticomon.com.ar, ivelis.sarachi@gmail.com
\end{abstract}

Received: June 09, 2017 | Published: June 26, 2017
Abbreviations: PTC, papillary thyroid carcinoma; US, ultrasound; FNA, fine-needle aspiration; E, elastography; SR, strain ratio; ROIs, regions of interest

\section{Introduction}

PTC is asymptomatic with a favorable prognosis, with overall 10year survival rates near to $95 \%$. Independent risk factors for recurrence are age at diagnosis (over 45years old), tumor size (more than 10 $\mathrm{mm}$ ), pathologic subtype, radioactive iodine therapy, extrathyroidal extension, and lymph node metastasis.

Best diagnosis imaging modality to evaluate thyroid nodule is the US. Marked hypoechogenicity, poorly defined margin, microcalcifications, and taller than wide, are the US features that can be used to predict malignant nodules and poor prognosis. Thyroid stiffness index obtained with $\mathrm{E}$ is useful in differentiating between malignant and benign thyroid nodules. It is possible measure tissue deformation in response to compression, what give us an idea of tissue stiffness. ${ }^{1,2}$

\section{Case presentation}

We report a case of PTC discovered by E. A 54years old female with a studied thyroid nodule during 5years, was performed US and E. The nodule was described as circumscribed, markedly hypoechoic, with no vessels, in thyroid left lobe. With a diameter of $9 \mathrm{~mm}$, stable during the time. E shown an inner area of stiffness ( $3 \mathrm{~mm}$ size) and an outer area softer. Thyroid homogeneous echogenicity, without evidence of another nodule. We performed FNA E-guided of stiffness area. The cytology reported compact clusters of follicular cells with moderate anisokaryosis, empty nuclei and papillary branches, with microcalcification, that indicated suspicion of papillary carcinoma (Bethesda V). At surgery cancer had spread to local lymph nodes. The patient was undergone completion thyroidectomy and received radioactive iodine treatment (Figure 1) (Figure 2).

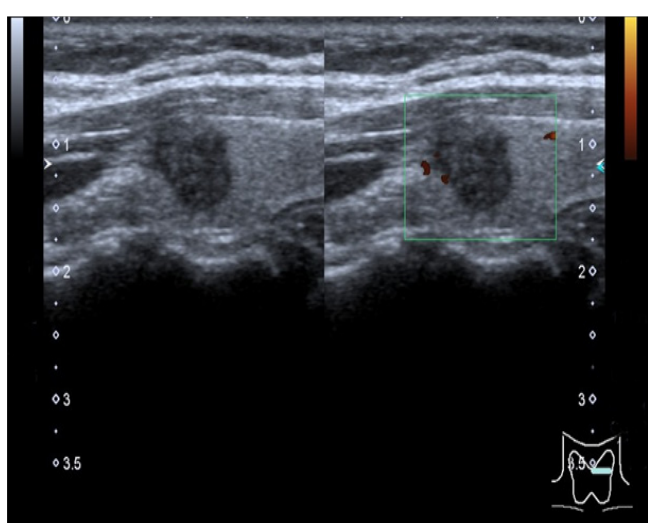

Figure I Gray scale (left) and power doppler (right) of thyroid nodule in the left lobule that shows no vascularization.

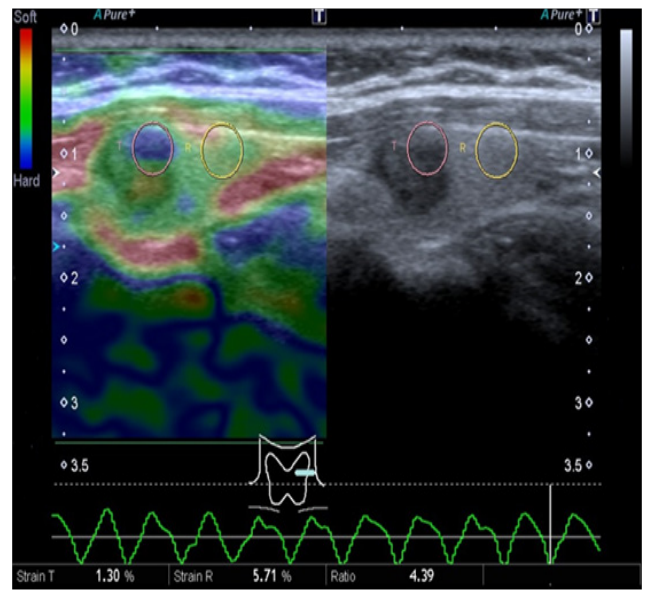

Figure 2 Elastogram (left) and gray scale (right) of thyroid nodule. Scale E represents stiffness areas with color blue, and softer with green and red. This E shows a stiffness inner area (with a $4.39 \mathrm{SR}$ ) with a softer outer area. 


\section{Discussion}

PTC is the most common type of all thyroid cancers (about $80 \%$ ), and can occur at any age. It tends to grow slowly and often affect lymph nodes in the neck. Has generally excellent prognosis, even if spread to the lymph nodes. At US, it is typically marked hypoechogenicity, with microcalcifications, poorly defined margin, and presence of intranodular and peripheral flow Doppler. The primary therapy for thyroid cancer is surgery. The extent of surgery for differentiated thyroid cancers will depend on the size of the tumor and if the tumor is confined to the thyroid. For very small cancers $(<10 \mathrm{~mm})$ that are confined to the thyroid, involving only one lobe and without evidence of lymph node involvement a simple lobectomy (removal of only the involved lobe) is considered sufficient. After surgery, most patients need to be on treatment with thyroid hormone for the rest of their life. If the cancer is large and if it has spread to lymph nodes, treatment with radioactive iodine can be used after the thyroid gland removed..$^{1-3}$

There are two types of E: semi-quantitative (strain E) and quantitative (shear-wave E). We use strain E. Strain E detects the local deformation under slight pressure and displays it as a relative value in comparison to the strain values of the different tissues within the region of interest. To acquire the elastographic images, compression is continuously applied by a transducer and followed by decompression. The elastic image is superimposed on the B-mode image, and tissue stiffness is displayed in a continuum of colors from red (soft tissue) to blue (hard tissue). We use Ueno scale. A score of 1 defined elasticity that is entirely soft in the nodule, 2 as mostly soft in the nodule, 3 as peripherally soft, 4 as entirely hard in the nodule, and 5 as hard in the area under consideration as well as the entire nodule. We chose particularly the valley area or the ascending part of one wave (compression). Two regions of interest (ROIs) are drawn over the target region and the adjacent reference region, respectively. Then, a strain ratio (SR) is automatically calculated, with a maximum value considered normal up to 2.45 . The likelihood of malignancy increases with an increase in the strain ratio. ${ }^{4}$ There are limiting factors that can affect the results of elastography, including nodule characteristics (calcifications and cystic components), the experience of the operator, and motion artifacts such as carotid artery pulsation. However, E optimizes the US imaging. In our case, the FNA of stiff area was E guided. The $\mathrm{E}$ is a promising technique to guided FNA and predict the preoperative malignancy risk.

\section{Acknowledgements}

I appreciate the support of the endocrinologist Greco Pascual, the radiologist Mon Guillermo and the engineer Claudio Forti.

\section{Conflict of interest}

Author declares that there is no conflict of interest.

\section{References}

1. Jun-Mei Xu, Xiao-Hong $\mathrm{Xu}$, Hui-Xiong Xu, et al. Conventional Us, Us elasticity imaging, and acoustic radiation Force impulse imaging for Prediction of Malignancy in Thyroid nodules. Radiology. 2014;272(2):577-586.

2. Soo-Yeon Kim, Jin Young Kwak, Eun-Kyung Kim, et al. Association of Preoperative Us Features and recurrence in Patients with classic Papillary Thyroid carcinoma. Radiology. 2015;277(2):574-583.

3. Dighe Manjiri, Bae Unmin, Richardson Michael L, et al. Differential Diagnosis of Thyroid Nodules with US Elastography Using Carotid Artery Pulsation. Radiology. 2008;248(2):662-669.

4. Barr Richard G, Nakashima Kazutaka, Amy Dominique, et al. WFUMB guidelines and recommendations for clinical use of Ultrasound Elastography: part 2; breast. Ultrasound in medicine and biology. 2015;41(5):1148-1160. 